https://doi.org/10.15407/ujpe65.8.737

\title{
TO THE 95th BIRTHDAY \\ OF DMYTRO VASYLYOVYCH VOLKOV (1925-1996)
}

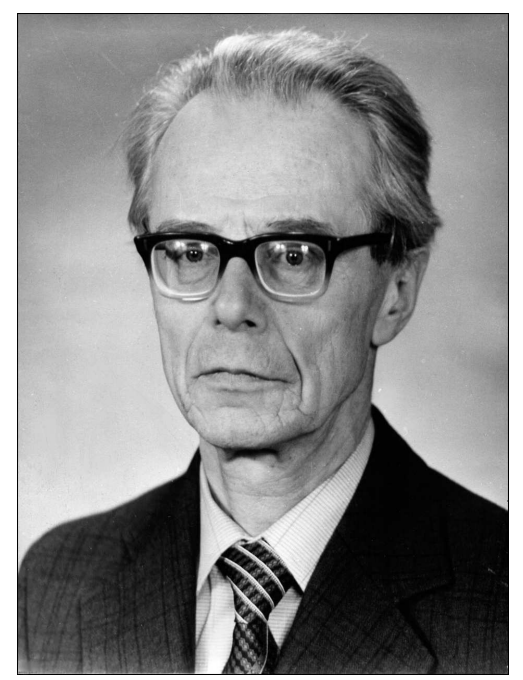

July 3, 2020 marked the 95th anniversary of the birth of the outstanding physicist-theorist Dmytro Vasylyovych Volkov, Academician of the National Academy of Sciences of Ukraine, Honored Worker of Science and Engineering of Ukraine.

In 1971-1972, D.V. Volkov (in Kharkiv) and Yu.A. Golfand together with E.P. Likhtman (in Moscow) discovered the supersymmetry. In 1973, Dmytro Vasylyovych discovered the gauge generalization of the supersymmetry, which was later coined the supergravity. The discoveries of the supersymmetry and supergravity became outstanding achievements in theoretical physics of the 20th century. Together with the concept of strings, they formed a basis of the modern approach to the Standard Model extension and the construction of a unified theory of elementaryparticle interactions.

The supersymmetry unifies bosons with fermions into multiplets and removes the prohibition on the non-trivial combinations of internal symmetry groups with the Poincaré group, which was dictated by the Coleman-Mandula theorem known in quantum field theory. The supersymmetric generalization of the Poincare group is accompanied with the expansion of the space-time with new fermionic coordi- nates. Dmytro Vasylyovych established that, in the case of spontaneous breaking of the Poincaré supergroup, those coordinates manifest themselves as massless fermion fields. Those fermions are generalizations of the Nambu-Goldstone bosons that arise in the theory of quasimean values of M.M. Bogolyubov owing to the spontaneous breaking of internal symmetries.

The unification of the supersymmetry with the general theory of relativity led Dmytro Vasylyovych to a deep generalization of the fundamentals of gravitation theory based on applying the concept of a graviton, the spin 2 boson gauge field. D.V. Volkov introduced a new fermion gauge field, gravitino with spin $3 / 2$, into the theory of gravitation and built the world's first theory of supergravity. The new theory combined the interacting fields of gravitons and gravitinos into a single gauge supermultiplet $(2,3 / 2)$ and gave rise to the discovery of the supersymmetric Higgs effect, which accompanies the spontaneous breaking of the local supersymmetry. The theory of supergravity was further developed in the well-known works by Ferrara, Freedman, and van Nieuwenhuizen and by Deser and Zumino, which were published in 1976.

The wide recognition of the discovery of supergravity by Dmytro Vasylyovych was evidenced by his invitation in 1994 as an honored guest with the report "Supergravity before 1976" to the international conference on the history of original ideas and basic discoveries in particle physics of the 20th century (Erice, Italy). His last report, which was devoted to the generalized principle of action for strings and branes, D.V. Volkov made at the SUSY-95 conference in Paris. In honor of the outstanding contribution of D.V. Volkov to the development of particle physics, the organizers dedicated the proceedings of this conference to his memory.

The works by D.V. Volkov substantially defined the directions of further development of theoretical and experimental researches in high-energy physics at leading scientific centers. The search for new particles predicted by supersymmetry is one of the main areas 
of researches at the Large Hadron Collider, as well as at astrophysical observatories on the Earth and in space. For the discovery of supersymmetry and supergravity, D.V. Volkov was posthumously awarded the Walter Tirring Medal in 1997 and, together with his disciples, the State Prize of Ukraine in science and technology in 2009. In 2007, Presidium of the National Academy of Sciences of Ukraine established the D.V. Volkov Prize. Dmytro Vasylyovych himself was awarded numerous state awards.

Among the pioneering works of D.V. Volkov, it is worth noting the discovery of a new scheme for field quantization known as the parastatistics or GreenVolkov statistics, the discovery of the origin of the connection between the poles in the amplitudes of the nucleon-nucleon and nucleon-antinucleon scattering known as the "Regge pole conspiracy", the development of the method of phenomenological Lagrangians for the description of the spontaneous breaking of internal symmetries and its application to the description of magnons in magnetic materials as NambuGoldstone bosons, the solution of the problem of spontaneous vacuum transitions in the Veneziano and Neveu-Schwarz dual models, as well as some other known studies in the theory of superstrings and branes performed together with his disciples. Those works made an important contribution to the development of quantum field theory and elementary particle physics.

The scientific activity of Dmytro Vasylyovych was inseparably linked with the Kharkiv Institute of Physics and Technology, where he worked for 40 years and trained a pleiad of the physicist-theorists, whose works are known far beyond Ukraine.

The heavy fighting on the Karelian and Far Eastern fronts during World War II, for which Dmytro Vasylyovych was awarded military awards, built his character and formed his attitude to life. He was an inherently democratic and principled person intolerant to the violations of scientific ethics, the manifestations of bureaucracy, careerism, and injustice. In his life, Dmytro Vasylyovych was characterized by the kindness to people, modesty, unpretentiousness, and simplicity in communication. He tried to help everybody who asked for his aid. Everyone who knew Dmytro Vasylyovych marked the fundamental character of his approach to solving the life problems, the depth of his intuition, and his ability to immediately grasp the essence of the problem and find an unexpected solution. The communication with him on the burning life and scientific issues revived the optimism and a belief that a lot of problems can be solved by demonstrating a respect and a reasonable attitude to people and events. The name of D.V. Volkov, an outstanding scientist and a wonderful man, will forever remain in the memory and hearts of his colleagues and disciples.

O.S. BAKAI, V.G. BAR'YAKHTAR, V.P. GUSYNIN, O.O. ZHELTUKHIN, A.G. ZAGORODNY, V.M. LOKTEV, S.V. PELETMINSKII, YU.O. SITENKO, YU.V. SLYUSARENKO, V.YU. STORIZHKO, D.V. UVAROV, M.F. SHULGA 